\title{
Culture of bullying in medicine starts at the top
}

\author{
Cite as: CMAJ 2018 December 10;190:E1459-60. doi: 10.1503/cmaj.109-5690
}

Posted on cmajnews.com on Nov. 20, 2018.

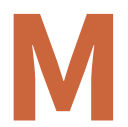

ore than a third of doctors in the United Kingdom say that bullying, undermining behaviour and harassment are problems in their workplaces, according to the British Medical Association (BMA). Workplace stress is part of the problem, but so are health care leaders who tolerate bullying down the line.

In a survey of 7887 doctors, $29 \%$ said bullying was "sometimes" a problem and $10 \%$ said it was "often" an issue. One in five had experienced bullying, undermining behaviour or harassment in the past year, but only $33 \%$ said they or a colleague had reported the incident.

Asked about the main causes of bullying, $65 \%$ of doctors said people are under pressure, and $58 \%$ said it was difficult to challenge because "the behaviour comes from the top." Half said people who are bullied or harassed are too afraid to speak up. Two in five attributed the problem to managers lacking the commitment (43\%) or training (39\%) to deal with bullying. Many doctors also reported "bullying down the line" with senior staff offloading pressures onto those next in line.

Similar problems have been reported in Canada. In a recent survey, more than three-quarters of medical residents surveyed said they had experienced some form of harassment or intimidation in the preceding year.

The BMA is calling on the UK's National Health Service (NHS) to improve resolution processes and create a more inclusive culture where people feel safe to speak up about bullying. According to the BMA, it is "especially important" for managers, leaders, teachers and supervisors to identify and address bullying. "Encouraging individuals to speak up and report bullying and harassment will not be effective if complaints are not taken seriously."

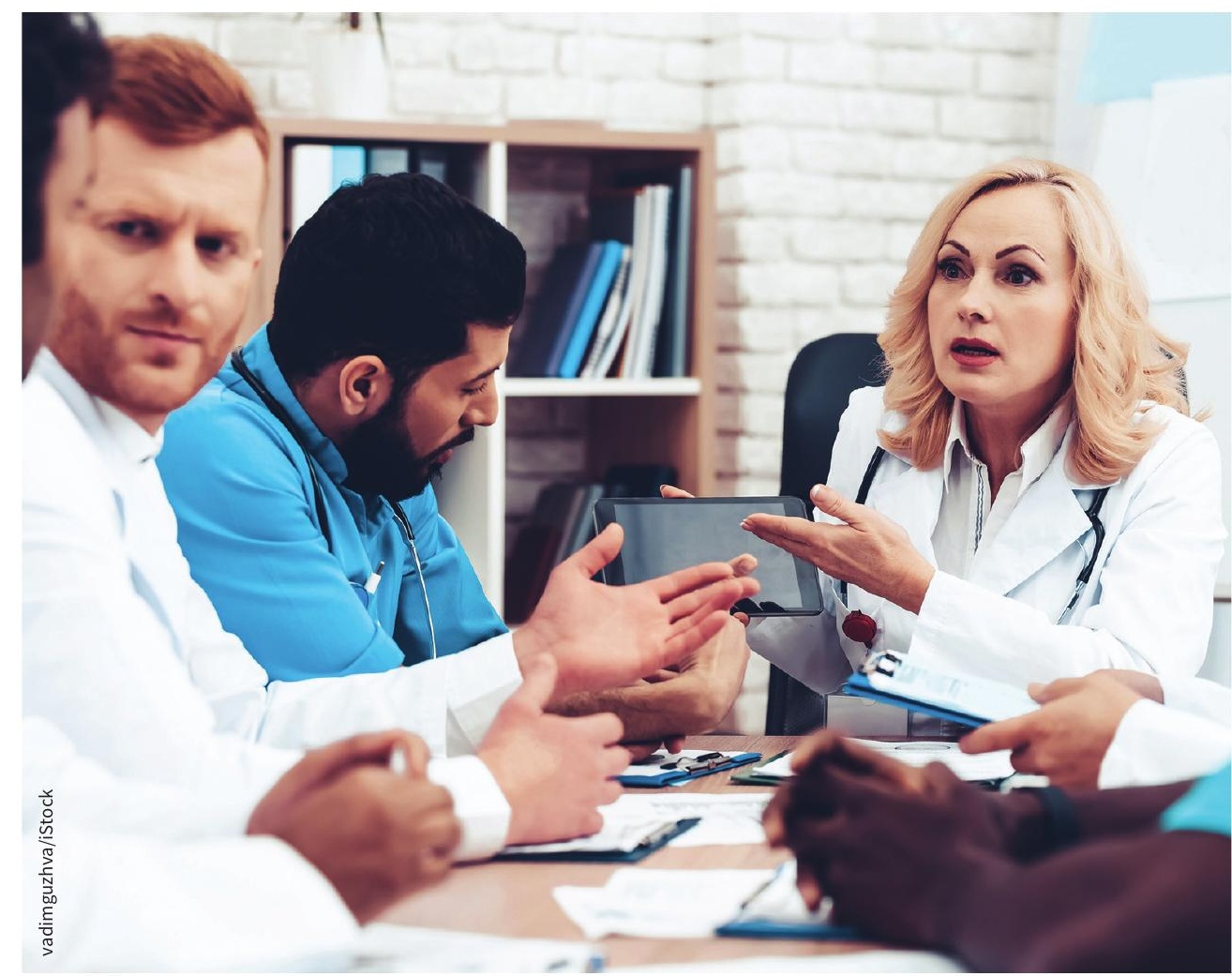

Health leaders set the tone on bullying, reports the British Medical Association.

In one case, a trainee doctor who was allegedly pinned to a wall by the throat by a consultant was told by other consultants "not to make trouble," as it might affect the ability to get references. Another consultant told the BMA, "among senior doctors, the culture is still very much that you suck it up."

Ignoring such incidents comes at a heavy cost to both individuals and organizations, the BMA warns. Some people who reported being bullied say it destroyed their confidence and caused lasting harm to their careers. Even bystanders are more likely to take time off or want to change jobs.

In addition to increased absences and turnover among health workers, "the conse- quences for patient care and safety are serious," says Anthea Mowat, BMA chair of the representative body. Where bullying is common, "staff are afraid to raise legitimate concerns about patient care or safety."

According to the BMA, "a change of leadership style is needed in the NHS." Efforts to address bullying and harassment "should not simply exhort staff to behave better." The NHS must also tackle how heavy workloads, staff shortages, poor technology and "command and control" leadership are contributing to the problem. This includes providing training and support for managers and educators who often struggle to "draw the line between managing performance and bullying." 
The BMA also recommends that the NHS "implement existing policies and procedures better." Complaints should not be "dismissed out of hand" and resolution processes must not "drag on unnecessarily." Formal investigations typically take six months to a year to resolve, and some carry on "much longer." As a result, some people avoid reporting bullying and harassment or allow the situation to drag on for months or years before they reach a breaking point.

According to the BMA, health organizations should ensure there are alternative means of resolving bullying complaints, such as mediation, and there should be someone people can contact for informal support. Managers, senior physicians and human resources staff should also intervene earlier to address one-off incidents of unprofessional behaviour, such as rudeness. Waiting to see if conflicts escalate leads to problems that are "harder to resolve and cause more harm in the long run." An upcoming national conference will provide practical guidance on tackling bullying in the NHS.

Lauren Vogel, CMAJ 